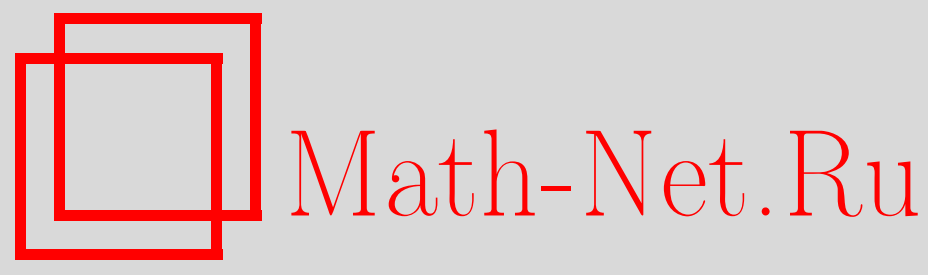

Д. П. Ильютко, Локально минимальные сети в $N$ нормированных пространствах, Матем. заметки, 2003, том 74, выпуск 5, 656-668

DOI: https://doi.org/10.4213/mzm298

Использование Общероссийского математического портала Math-Net.Ru подразумевает, что вы прочитали и согласны с пользовательским соглашением http://www.mathnet.ru/rus/agreement

Параметры загрузки:

IP : 35.173 .137 .237

26 апреля 2023 г., 16:41:46

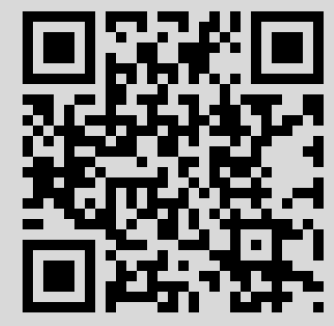




\title{
ЛОКАЛЬНО МИНИМАЛЬНЫЕ СЕТИ В $N$-НОРМИРОВАННЫХ ПРОСТРАНСТВАХ
}

\section{Д. П. Ильютко}

\begin{abstract}
В настоящей статье мы дадим полную классификацию локально минимальных сетей в нормированных пространствах $\left(\mathbb{R}^{2}, \rho\right)$, где единичная окружность $\Sigma=\left\{x \in \mathbb{R}^{2} \mid\right.$ $\rho(x)=1\}$ для нормы $\rho$ совпадает с правильньм $m$-угольником, $m=2 n$, вписанньп в евк лидову единичную окружность $S^{1}$.

Библиограффия: 9 названий.
\end{abstract}

1. Введение и постановка задачи. Настоящая работа посвящена описанию локальной структуры линейных кратчайших сетей в пространстве $\left(\mathbb{R}^{2}, \rho\right)$, где единичная окружность $\Sigma=\left\{x \in \mathbb{R}^{2} \mid \rho(x)=1\right\}$ для нормы $\rho$ совпадает с правильньм $m$-угольником, $m=2 n$, вписанным в евклидову единичную окружность $S^{1}$ так, что пара его вершин лежит на оси абсцисс.

С точки зрения римановой геометрии локально минимальные сети являются естественньм обобщением обычных геодезических. Напомним, что геодезическая, соединяющая пару фиксированных точек риманова многообразия, обладает следующим определяющим свойством: каждьй ее фрагмент между достаточно близкими точками $A$ и $B$ является кратчайшей кривой среди всех кривых, соединяюших $A$ и $B$. Предположим, что фиксировано не две точки риманова многообразия $W$, а некоторое конечное его подмножество $M$, состоящее из большего числа точек. Чтобы обобщить на этот случай понятие геодезических, естественно перейти от кривых к рассмотрению связньг одномерных континуумов - сетей. Сеть, затягивающая множество $M \subset W$, назьвается абсолютно минимальной, если она имеет наименьшую возможную длину, и локально минимальной, если каждый достаточно мальй ее фрагмент имеет наименьшую возможную длину, т.е. является абсолютно минимальной сетью. В этом смысле естественно говорить о локально минимальных сетях как о "разветвленных геодезических".

Понятие локально минимальной сети впервые неявным образом появилось при изучении следующей классической задачи, известной в литературе как проблема Штейнера в случае евклидовой длины: среди всех сетей (связных одномерных континуумов), затягивающих данное конечное множество $M$ точек плоскости, найти сеть наименьией длины.

При подготовке данной работы автор ползовался частичной поддержкой Российского фонда фундаментальных исследований, проекты № 01-01-00583 и 01-15-99268, и Euler Program. 
Эта задача была названа “проблемой Штейнера" в замечательной книге Ричарда Куранта и Герберта Е. Роббинса “ Что такое математика?" в честь Якоба Штейнеpa (Jacob Steiner, 1796-1863), швейцарского математика, профессора Берлинского университета. Именно после появления этой очень популярной книги интерес к абсолютно минимальным сетям разгорелся с новой силой, и терминология, предложенная Курантом и Роббинсом, стала общепринятой.

Исторический обзор, посвященньй проблеме Штейнера, см. в [1]-[4].

Отметим, что проблемой Штейнера занимались многие известные математики, такие как Винтер, Гилберт, Гильдебрандт, Грехем, Гэри, Джонсон, Ду, Кокейн, Мантуров, Мелзак, Морган, Поллак, Рубинштейн, Смит, Томас, Фоменко, Ханан, Хванг, Цислик и другие. Одна из причин этого неослабевающего интереса специалистов к минимальньм сетям состоит в том, что у проблемы Штейнера имеется много различных интерпретаций и приложений. Например, заданное конечное множество $M$ можно интерпретировать как набор конечных (терминальных) пунктов. Если, например, терминальные пункты - города, которые требуется соединить сетью дорог, то в этом случае минимальная сеть - это самая дешевая транспортная система, обеспечивающая коммуникации между данньми конечньми пунктами. Здесь естественно предполагается, что стоимость коммуникаций пропорциональна их длине.

Первые работы, посвященные изучению кратчайших сетей в смысле нормированной длины, появились в 60-е годы (см. [5]) в связи с бурным развитием электроники и робототехники. Интерес возник в связи с тем, что проводники на печатных платах имеют, как правило, вид ломаных линий, составленных из горизонтальных и вертикальных отрезков. По-видимому, первое систематическое исследование кратчайших сетей в смысле манхетенской длины (так назьваемых кратчайших прямоугольных деревьев) было предпринято в 1966 году Ханном [6], которьй описал несколько важных общих геометрических свойств таких сетей.

Спустя 10 лет Хванг [7] описал возможную структуру кратчайших прямоугольных деревьев в предположении, что данное множество затягивается хотя бы одним невырожденным кратчайшим деревом $\Gamma_{0}$. Последнее означает, что степень всех граничных вершин в дереве $\Gamma_{0}$ равна 1 . Однако эффективньй алгоритм, строящий кратчайшеепрямоугольное дерево, найти не удалось. Объяснение этому было дано в 1977 году Гэри и Джонсоном [8], которые показали, что задача поиска кратчайшего прямоугольного дерева является $N P$-полной, т.е. скорее всего не существует полиномиального алгоритма решения этой задачи. Этот факт делает изучение ограничений на структуру кратчайших сетей еще более актуальной.

В настоящей работе получено полное описание локальной структуры кратчайших сетей в пространстве $\left(\mathbb{R}^{2}, \rho\right)$, где единичная окружность $\Sigma=\left\{x \in \mathbb{R}^{2} \mid \rho(x)=1\right\}$ для нормы $\rho$ совпадает с правильным $m$-угольником, $m=2 n$, вписанным в евклидову единичную окружность $S^{1}$ так, что пара его вершин лежит на оси абсцисс.

Введем некоторые понятия.

ОПРЕДЕЛЕнИЕ. Линейной сетью назьвается сеть в $\mathbb{R}^{2}$, все ребра которой-прямолинейные отрезки (возможно вырожденные). Если все ребра не вырожденные, то сеть назьвается вложенной.

ОПРЕДЕЛЕниЕ. Сеть, затягивающая некоторое множество, называется кратчай- 
шей, если ее длина не превосходит длины любой сети, затягивающей данное множество.

ОПРЕДЕЛЕНИЕ. Сеть назьвается локально минимальной, если любой достаточно малый фрагмент сети является кратчайшим.

Пронумеруем все вершины $m$-угольника $\Sigma$ против часовой стрелки, начиная с нуля, г де вершина с номером $i$ имеет координаты $(\cos (\pi i / n), \sin (\pi i / n))$, и обозначим эту вершину через $P_{i}$. Тем самым для каждой стороны $m$-угольника $\Sigma$ заданы начало и конец этой стороны.

Рассмотрим ребро $\gamma$ сети $Г$. В теореме о локальной структуре изучаемых сетей положение ребер будет определяться неоднозначно. Поэтому для удобства формулирования теоремы мы будем характеризовать положения ребер следуюшими пятью типами. Будем говорить, что ребро ү сети имеет точечны й тuп, если направление этого ребра может приходить только в вершину $m$-угольника $\Sigma$. Будем писать type $e_{e}(\gamma)=*$, и для данного ребра обозначим через $\chi(\gamma)$ вершину из $\Sigma$, соответствующую направлению ребра $\gamma$. Будем говорить, что ребро $\gamma$ сети имеет неточечный тип, если направление этого ребра может лежать на стороне $m$-угольника $\Sigma$, причем граничные вершины стороны могут быть включены, а могут и нет. Будем писать type $e_{e}(\gamma)=[],(),[)$ и (] в зависимости от того включена граничная вершина стороны (соответствует "[" или “]”) или нет (соответствует "(" или “)”), и для этих ребер определим $\chi(\gamma)$ как сторону $m$-угольника $\Sigma$, содержащую направление этого ребра.

Для любых подмножеств $A$ и $B$ из $m$-угольника $\Sigma$ обозначим через $\alpha(A, B)$ точную нижнюю грань углов между радиус-векторами точек $x \in A$ и $y \in B$. Пусть $\gamma_{1}$ и $\gamma_{2}-$ произвольные ребра сети Г. Будем говорить, что пара $\left(\gamma_{1}, \gamma_{2}\right)$ uмeет nогрешность $k$, и будем писать fall $\left(\gamma_{1}, \gamma_{2}\right)=k$, если $\alpha\left(\chi\left(\gamma_{1}\right), \chi\left(\gamma_{2}\right)\right)=2 \pi / 3-k \pi / 3 n$.

Введем понятие типа вершины $x$, и обозначим его через type $v_{v}(x)$. Пусть вершина $x$ сети $Г$ имеет степень $l$, и $\gamma_{1}, \ldots, \gamma_{l}$ - выходящие из этой вершины ребра, направления которых являются последовательными при обходе начала координат в положительном направлении от оси абсцисс. Положим type $v(x)=\left(\operatorname{type}_{e}\left(\gamma_{1}\right), \ldots, \operatorname{type}_{e}\left(\gamma_{l}\right) ;\right.$ fall $\left(\gamma_{1}, \gamma_{2}\right)$, fall $\left(\gamma_{2}, \gamma_{3}\right), \ldots$, fall $\left.\left(\gamma_{l-1}, \gamma_{l}\right)\right)$.

ОСновнАЯ ТЕОРЕМА. Влохенная линейная сеть Г с некоторой границей в пространстве $\left(\mathbb{R}^{2}, \rho\right)$ является локально минимальной, если и только если вериинь сети Г имеют степень, которая не превосходит 6, и следующие типь ( тью до поворота):

1) если степень вершины $x$ сети Г равна 1, то эта вершина граничная и $\operatorname{type}_{v}(x)=\{[]\}$

2) если степень вершины $x$ сети Г равна 2, то

(a) для внутренней вериины tуре ${ }_{v}(x)=\{[],[] ; 3-n\}$;

(b) для граничной вершинь $п р и 2 n \equiv k(\bmod 3), \quad k=1,2,3$, имеем $\operatorname{type}_{v}(x)=\{[],[] ; l\}$, причем $3-n \leqslant l \leqslant k ;$

3) если степень вершины $x$ сети Г равна 3 , то

(a) $n p u 2 n \equiv 0(\bmod 3)$ имеем $\operatorname{type}_{v}(x)=\{[],[],[; ; 3,3\}$;

(b) $n p u 2 n \equiv k(\bmod 3), k=1,2$ имеем $\operatorname{type}_{v}(x)=\{*,[], * ; k, k\}$;

(c) если вершина граничная $u 2 n \equiv 0(\bmod 3), \operatorname{mo~type~}_{v}(x)=\{[],[], * ; 0,3\}$;

(d) если вершина граничная $u 2 n \equiv 2(\bmod 3), \operatorname{mo~type~}_{v}(x)=\{[],[], * ; 2,2\}$;

4) если степень вершины $x$ сети Г равна 4, то $m=4,6,8$ или $12, u$ 
(a) $п р и т=4,8$ или 12 имеем type $_{v}(x)=\{*, *, *, * ; m / 4, m / 4, m / 4\}$;

(b) $n р и ~ m=6$ имеем type $v(x)=\{*,[], *,[] ; 3,3,3\}$;

(c) если вершина граничная и $m=6$, mo type $_{v}(x)=\{[],[], *, * ; 3,3,3\}$;

5) если степень вершины $x$ сети Г равна 5 или 6, то эта вериина - граничная, $m=6, u$

(a) если степень вершины равна 5, то туре ${ }_{v}(x)=\{[], *, *, *, * ; 3,3,3,3\}$;

(b) если степень вериины равна 6, mo type $_{v}(x)=\{*, *, *, *, *, * ; 3,3,3,3,3\}$.

Автор благодарен профессору А. О. Иванову и профессору А. А. Тужилину за постоянное внимание к работе.

2. Предварительные сведения. Напомним некоторые важные определения, которые нам понадобятся в дальнейших рассуждениях.

ОПРЕДЕЛЕНИЕ. Топологическим графом $G$ называется топологическое пространство, полученное из конечной совокупности отрезков $\left\{I_{\alpha}\right\}$ некоторой склейкой по их концам. Пусть $\pi: \sqcup_{\alpha} I_{\alpha} \rightarrow G$-каноническая проекция. Образы внутренностей отрезков $I_{\alpha}$ при отображении $\pi$ назьваются ребрами графа $G$, а $\pi$-образы конщевых точек отрезков $I_{\alpha}$ - вершинами. Граф $G$ связен, если он связен как топологическое пространство.

Предположим, что в графе $G$ выделено некоторое подмножество $B$ множества его вершин. Такой граф $G$ будем назьвать графом $с$ границей $\partial G=B$. Вершины из $\partial G$ будем назьвать граничными или неподвижными, а все остальные вершины - внутренними или подвижными. Ребра графа, инцидентные граничным вершинам, также назовем граничными, а ребро, не инцидентное никакой граничной вершине, назовем внутреннuм.

Пусть $G$ - произвольньй граф с гранищей $\partial G$ (возможно, пустой), и $P \in G$ - некоторая его точка. Допустимой окрестностью $U \subset G$ точки $P$ графа $G$ назьвается замькание связной окрестности этой точки, не содержащее вершин графа $G$, отличных от $P$, если $P$ - вершина. Наделим окрестность $U$ структурой графа, объявив вершинами все точки из $\partial U \cup\{P\}$, а ребрами - внутренности отрезков в $U$, соединяющих эти точки. Полученную звезду обозначим через $G_{U}$ и будем называть локальнылм графом $c$ иентром в точке $P$. Определим каноническую гранииу $\partial G_{U}$ локального графа $G_{U}$, включив в нее все вершины из $\partial U$, а также вершину $P$, если $P$ - граничная вершина графа $G$. Другими словами, $\partial G_{U}=(\partial G \cap U) \cup(G \cap \partial U)$.

ОПРЕДЕЛЕНИЕ. Пусть $G$ - произвольньй связньй топологический граф, и $\partial G-$ его гранища. Параметрической сетью топологии $G$ на топологическом пространстве $X$ назьвается непрерывное отображение $\Gamma$ из $G$ в $X$. Топологический граф в этом случае назьвается параметризующим графом параметрической сети Г или ее топологией.

ОПРЕДЕЛЕниЕ. Ограничение отображения Г на вершины, ребра, границу, связньй подграф параметризующего графа, локальный граф называются соответственно вершинами, ребрами, граничей, подсетью, локальной сетью параметрической сети Г.

ОПРЕДЕЛЕНИЕ. Пусть $X$ - гладкое многообразие. Параметрическая сеть $\Gamma: G \rightarrow X$ называется гладкой (регулярной, кусочно-гладкой, кусочно-регулярной), если ограничение отображения $\Gamma$ на замыкание каждого ребра графа $G$ является таковым. 
ОпРЕДЕЛЕНИЕ. Кусочно-регулярные параметрические сети без кратных ребер и петель называются погружсенными. Погруженную параметрическую сеть Г назовем $\boldsymbol{8 л о -}$ женной, если отображение Г взаимно однозначно с образом.

ОПРеДЕЛЕниЕ. Ребро $\gamma$ сети Г назьвается точечным, если оно - отображение в точку. Ребро $\gamma$ сети Г называется квазирегулярным , если оно или регулярно, или точечно. Сеть Г квазирегулярна, если все ее ребра квазирегулярны.

ОПРЕДЕЛЕНИЕ. Линейной сетью назьвается сеть в $\mathbb{R}^{n}$, все ребра которой-прямолинейные отрезки (возможно вырожденные).

ОПРЕДЕЛЕНИЕ. Точечная компонента сети Г - это связная компонента множества точечных ребер сети. Приведенная компонента сети $Г$ - это или ее точечная компонента, или вершина, которая не принадлежит точечным компонентам.

Пусть $\Gamma: G \rightarrow X$ - произвольная параметрическая сеть, и $I=[a, b]-$ некоторьй отрезок.

ОПрЕДЕЛЕНИЕ. Непрерьвное отображение $\Psi: G \times I \rightarrow X$ такое, что для всех $g \in G$ имеет место равенство $\Psi(g, a)=\Gamma(g)$, назьвается деформаиией параметрической се$m u$ Г. Если исходная параметрическая сеть $Г$ является гладкой (регулярной, кусочно-гладкой, кусочно-регулярной), то мы будем предполагать, что каждая параметрическая сеть $\Psi(\cdot, t)=\Gamma_{t}$ является таковой, и что для каждого замыкания $\bar{e}$ ребра еграфа $G$ ограничение отображения $\Psi$ на $\bar{e} \times I$ является гладким (для регулярных сетей) или кусочно-гладким (для кусочно-гладких или кусочно-регулярных сетей).

Пусть $H$ - произвольньй подграф в топологическом графе $G$. Обозначим через $G / H$ топологическое пространство, полученное из $G$ отождествлением точек, попавших в связные компоненты графа $H$. Пространство $G / H$ наделяется естественной структурой топологического графа. Граф $G / H$ назьвается фактор-графом графа $G$ по подграфу $H$. Будем говорить, что граф $G_{2}$ может быть спроектирован на граф $G_{1}$, и будем писать $\pi: G_{2} \rightarrow G_{1}$, если существует $H \subset G_{2}$ такое, что $G_{1}=G_{2} / H$. Аналогично определяется проекция $G_{1}$ на $G_{2}$.

Пусть $\Gamma_{1}: G_{1} \rightarrow X$ и $\Gamma_{2}: G_{2} \rightarrow X$-произвольные квазирегулярные параметрические сети.

ОПРЕДЕЛЕНИЕ. Будем говорить, что параметрическая сеть $\Gamma_{2}$ может быть спроектирована на $\Gamma_{1}$, если существует проекция $\pi: G_{2} \rightarrow G_{1}$ такая, что $\Gamma_{2}=\Gamma_{1} \circ \pi$.

Пусть $Г$ и $\Gamma^{\prime}$ - произвольные параметрические сети, причем $\Gamma^{\prime}$ может быть спроектирована на $\Gamma$.

ОПРЕДЕЛЕниЕ. Произвольную деформацию сети $\Gamma^{\prime}$ назовем деформацией $c$ расщеплением сети Г. При этом сеть $\Gamma^{\prime}$ будем называть типом такого расщепления.

3. Локально минимальные сети. Пусть $X$ - конечномерное линейное пространство с некоторой нормой $\|\cdot\|$ и $\gamma:[a, b] \rightarrow X-$ некоторая непрерывная кривая. Кривая $\gamma$ называется измеримой, если существует предел $l(\gamma)$ длин ломаных, вписанных в эту кривую. Число $l(\gamma)$ называется в этом случае длиной кривой $\gamma$. Если кривая $\gamma$ кусочно-гладкая, то она измерима и

$$
l(\gamma)=\int_{a}^{b}\|\dot{\gamma}(t)\| d t
$$


Пусть $\Gamma$ - произвольная кусочно-гладкая сеть на $X$. Тогда длиной $l(\Gamma)$ сетu $\Gamma$ назовем сумму длин ее ребер.

ОПРЕДЕЛЕНИЕ. Сеть Г назьвается критической или әкстремальной, если для любой неподвижной на границе деформации $\Gamma_{t}^{\prime}, t \in[0,1]$, где $\Gamma_{t=0}^{\prime}=\Gamma^{\prime}-$ произвольный тип расщепления сети $\Gamma$, вьполнено соотношение

$$
\left.\frac{d}{d t}\right|_{t=0+} l\left(\Gamma_{t}^{\prime}\right) \geqslant 0 .
$$

ОПРЕДЕЛЕНИЕ. Сеть Г назьвается слабо критической или слабо әкстремальной, если для любой неподвижной на границе деформации (без расщепления) $\Gamma_{t}, t \in[0,1]$, где $\Gamma_{t=0}=\Gamma$, вьполнено соотношение

$$
\left.\frac{d}{d t}\right|_{t=0+} l\left(\Gamma_{t}\right) \geqslant 0 .
$$

ОПРЕДЕЛЕНИЕ. Сеть Г назьвается локально әкстремальной, если любой достаточно мальй фрагмент сети является экстремальным.

ОПРЕДЕЛЕНИЕ. Сеть Г назьвается слабо локально әкстремальной, если любой достаточно малый фрагмент сети является слабо экстремальным.

ОПРЕДЕЛЕниЕ. Сеть, затягивающая некоторое множество, называется кратчайшей, если ее длина не превосходит длины любой сети, затягивающей данное множество.

ОПРЕДЕЛЕНИЕ. Сеть назьвается локально минимальной, если любой достаточно малый фрагмент сети является кратчайшим.

Нетрудно доказать следующее утверждение.

УТВЕРЖДЕНИЕ 3.1. Вложсенная сеть локально минимальна тогда и только тогда, когда она является локально әкстремальной.

ОПРЕДЕЛЕНИЕ. Субградиентом вьпуклой вниз функции $F: \mathbb{R}^{n} \rightarrow \mathbb{R}$ в точке $x \in \mathbb{R}^{n}$ назьвается такой ковектор $\xi \in T_{x}^{*} \mathbb{R}^{n}$, что

$$
\xi(y-x) \leqslant F(y)-F(x)
$$

для всех $y \in \mathbb{R}^{n}$.

Далее, если $X \subset \mathbb{R}^{n}$ - вьпуклая поверхность и $x \in X$ - произвольная ее точка, то проходящая через $x$ гиперплоскость П назьвается опорной плоскостью поверхности $X$ в точке $x$, если $X$ лежит в одном из замкнутых полупространств, ограниченных П. Нормаль к опорной гиперплоскости, направленную в то из ограниченных этой гиперплоскостью полупространств, внутренность которого не пересекается с $X$, назовем внешней нормалью к поверхности $X$ в точке $x$. Множество $N_{x} X$ всех внешних нормалей к поверхности $X$ в точке $x$ называется нормальным конусом.

При стандартном отождествлении пространств $T_{x}^{*} \mathbb{R}^{n}$ и $T_{x} \mathbb{R}^{n}$, субградиентное множество $S_{F}(x)$ выпуклой функиии $F$ в точке $x$, т.е. множество всех субградиентов функции $F$ в точке $x$, является непустым выпуклым ограниченным подмножеством нормального конуса в точке $x$ к поверхности уровня этой функции, проходящей через $x$. При этом, функция $F$ дифференцируема в $x$, если и только если множество $S_{F}(x)$ состоит из одной точки, совпадающей в этом случае с градиентом функции $F$. Если $F=\rho-$ некоторая норма, то легко доказьвается следующий результат, см. [9]. 
ПРЕДЛОЖЕНИЕ 3.1. СУбградиентное множество $S_{\rho}(x)$ в точке $x \neq 0$ совпадает с мнохсеством всех внешних нормалей единичной конормы $к$ поверхности уровня нормы $\rho$, проходящей через $x$.

Пусть $x \in \mathbb{R}^{2}$ - произвольная точка, и $\eta \in T_{x} \mathbb{R}^{2} \approx \mathbb{R}^{2}$ - любой вектор. Через $p(\eta)$ будем обозначать субградиент функции $\rho$ в точке $x$, причем, если $\eta$ и $x \neq 0$ коллинеарны, то $p(\eta)$ - любой такой ковектор, а если $\eta$ и $x \neq 0$ линейно независимы, то $p(\eta)$ удовлетворяет следующему дополнительному условию: вектор $p(\eta)$ ортогонален, в смысле евклидова скалярного произведения, радиальной проекции луча $x+t \eta, t \geqslant 0$, на окружность $\Sigma=\left\{z \in \mathbb{R}^{2} \mid \rho(z)=1\right\}$.

Пусть $\Gamma: G \rightarrow \mathbb{R}^{2}$ - произвольная квазирегулярная параметрическая сеть. Для каждой приведенной компоненты $H \subset \Gamma$ обозначим через $E_{H}$ и $V_{H}$ множество ребер и вершин сети $H$. Через $\partial H$ обозначим множество вершин из $H$, являюшихся граничными в сети $\Gamma$. Через $\bar{\partial} H$ обозначим множество вершин из $H$, инцидентных регулярным ребрам сети Г. Далее, для каждой вершины $x \in \bar{\partial} H$ обозначим через $N_{H}(x)$ множество регулярных ребер сети $\Gamma$, инцидентных $x$. Если $\gamma$ - произвольное ребро сети, то обозначим через $\partial \gamma$ пару его концевых вершин. Положим $\rho(\{A, B\})=\rho(B-A)$.

Для произвольного регулярного ребра $\gamma=[x, y]$ и произвольных векторов $\eta_{1}, \eta_{2} \in$ $\mathbb{R}^{2}$ положим $p_{x}\left(\gamma, \eta_{1}, \eta_{2}\right)$ равным $\rho$-импульсу $p_{x y}\left(\eta_{1}, \eta_{2}\right)$, которьй определяется равенством $p_{x y}\left(\eta_{1}, \eta_{2}\right)=p_{1}\left(\eta_{1}-\eta_{2}\right)=-p_{2}\left(\eta_{2}-\eta_{1}\right)$, где $p_{i}(\eta)-$ субградиент $p(\eta)$, вычисленный в точке $n_{i}=(-1)^{i}(y-x) /\|y-x\|$. Здесь $n_{i}$ - внешняя нормаль в смысле евклидова скалярного произведения к отрезку $[x, y]$ в точке $y$, если $i=2$, и в точке $x$, если $i=1$.

Справедлива следующая теорема, доказанная Ивановьм и Тужилиным, см. [9].

ТЕОремА 3.1. Пусть $\left(\mathbb{R}^{2}, \rho\right)$ - нормированное пространство. Квазирегулярная параметрическая линейная сеть $\Gamma: G \rightarrow \mathbb{R}^{2}$ с границей $\beta$ является слабой әкстремалью функиионала нормированной длины, если и только если для каждого отобрахсения $\eta: V_{G} \rightarrow \mathbb{R}^{2}$, әде $\eta(\partial G)=0$, следуюшая сумма по всем приведенным компонентам $H$ сети Г неотрицательна:

$$
\sum_{H}\left\{\sum_{x \in \bar{\partial} H}\left\langle\sum_{\gamma \in N_{H}(x), \gamma=[x, y]} p_{x}(\gamma, \eta(x), \eta(y)), \eta(x)\right\rangle+\sum_{\gamma \in E_{H}} \rho\left(\left.\eta\right|_{\partial \gamma}\right)\right\} \geqslant 0 .
$$

Из утверждения 3.1 следует, что для описания локальной структуры кратчайшей сети достаточно описать локальную структуру экстремальной сети. Отметим, что проверка экстремальности сети может быть сведена к проверке (с помощью теоремы 3.1) слабой экстремальности бесконечного числа параметрических сетей - различных типов расшеплений исходной сети. Тем не менее, оказьвается, эту проверку достаточно провести лишь для конечного числа типов расщепления.

Построим набор представителей, из слабой экстремальности которых вытекает экстремальность сети. Пусть $\Gamma$ - параметрическая сеть, $H$ - некоторая ее приведенная компонента, и $\bar{\Gamma}=\Gamma / H-$ сеть, полученная из $\Gamma$ факторизацией по $H$. Обозначим через $\pi: \Gamma \rightarrow \bar{\Gamma}$ каноническую проекцию, и пусть $x=\pi(H)$. Прообраз произвольной локальной сети $\bar{\Gamma}_{\text {loc }}(x) \subset \bar{\Gamma}$ для $\bar{\Gamma}$ с центром в $x$ при отображении $\pi$ назовем сильно локальной сетью приведенной компоненты $H$.

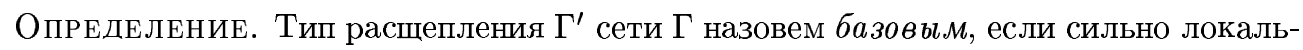
ная сеть $T$ каждой его вырожденной компоненты $H$ является бинарным деревом. При 
этом, если $H$ соответствует внутренней вершине сети $\Gamma^{\prime}$, то все вершины степени 1 дерева $T$ не принадлежат $H$. Если же $H$ соответствует граничной вершине $\Gamma^{\prime}$, то $H$ содержит ровно одну вершину $v$ дерева $T$ степени 1 , причем в этом случае $\partial H=\{v\}$.

Отметим, что у каждой вложенной сети Г имеется лишь конечное (не нулевое) число базовых типов расщепления.

Справедлива следующая теорема, доказанная Ивановьм и Тужилиным, см. [9].

ТЕОРемА 3.2. Вложенная линейная сеть Г представляет собой экстремаль функиионала нормированной длины в пространстве $\left(\mathbb{R}^{2}, \rho\right)$, если и только если каждый базовый тип расщепления $\Gamma^{\prime}$ сети Г является слабо әкстремальной параметрической сетью.

Имеет место следующее утверждение.

УТВЕРЖДЕНИЕ 3.2. Пусть Г - вложенная линейная сеть, все ребра которой отрезки, соединяющие начало координат $O$ с точками из $\Sigma$, причем все вершинь сети $\Gamma$, лежащие на т-угольнике $\Sigma$, являются граничными (вершина $O$ сети $Г$ может быть как граничной, так и внутренней). Предположим, что степень вериины $О$ больше 1 и сеть Г - экстремаль функиионала нормированной длины $в$ пространстве $\left(\mathbb{R}^{2}, \rho\right)$. Тогда

1) если $m=6$ и вершина $O$ сети Г внутренняя, то степень вериины $O$ сети Г меньше или равна 4 ;

2) если $m=6$ и вершина $O$ сети Г граничная, то степень вершины $O$ сети Г меньше или равна 6 ;

3) если $m=4,8,12$, то степень вершины $O$ сети $Г$ меньше или равна 4;

4) в остальных случаях степень вершинь $O$ сети Г меньие или равна 3.

ДокАЗАтельство. Рассмотрим тип расщепления $\Gamma^{\prime}$ сети $\Gamma$ с единственной вырожденной компонентой $H$, образ которой при отображении $\Gamma^{\prime}$ совпадает с $O$. При этом, если $O$ - внутренняя вершина, то $H$ - ребро, соединяющее внутренние вершины $x$ и $y$, а если вершина $O$ граничная, то $H$ - путь $x y, y z$ такой, что $x$ и $y$ - внутренние вершины, а $z$-граничная вершина степени 1 . Более того, сеть $\Gamma^{\prime}$ выберем так, чтобы вершина $x$ была инцидентна ровно двум смежным невырожденным ребрам $\gamma_{1}$ и $\gamma_{2}$.

Напомним, что $P_{k}$ - вершина $m$-угольника $\Sigma$ с номером $k$. Рассмотрим ребро $\gamma_{1}$. Имеется две возможности: или $\gamma_{1}$ соединяет $O$ с некоторой вершиной $P_{i_{1}}$ из $m$-угольника $\Sigma$, или $\gamma_{1}$ соединяет $O$ с какой-нибудь точкой $P$, лежащей внутри интервала $\left(P_{i_{1}-1}, P_{i_{1}}\right)$. Аналогично, для ребра $\gamma_{2}$ имеется две возможности: или $\gamma_{2}=O P_{i_{2}}$ для некоторой вершины $P_{i_{2}}$ из $m$-угольника $\Sigma$, или $\gamma_{2}=O Q$ для какой-нибудь точки $Q$, лежашей внутри интервала $\left(P_{i_{2}}, P_{i_{2}+1}\right)$. Таким образом, имеется четыре случая в зависимости от расположения вершин из $\gamma_{1}$ и $\gamma_{2}$. Так как повороты сети Г вокруг начала координат $O$ на угол кратный $\pi / n$ не меняют экстремальные свойства сети $\Gamma$, без ограничения общности будем предполагать, что $n-1 \geqslant i_{2}>i_{1} \geqslant 0$. Для каждого случая рассмотрим деформацию сети $\Gamma^{\prime}$ в классе линейных сетей, при которой вершина $x$ равномерно движется по прямой со скоростью $\eta=(\cos (\pi j / n), \sin (\pi j / n))$, где $j=i_{1}+\left(i_{2}-i_{1}-1\right) / 2$, если разность $i_{2}-i_{1}$ нечетна, и $j=i_{1}+\left(i_{2}-i_{1}\right) / 2$, если $i_{2}-i_{1}$ четна. При этом вершина $y$ остается на месте. 
Так как линейная сеть Г представляет собой экстремаль функционала нормированной длины в пространстве $\left(\mathbb{R}^{2}, \rho\right)$, тип расщепления $\Gamma^{\prime}$ сети $\Gamma$ является слабой экстремалью, поэтому из теоремы 3.1 вытекает, что выполняется следующее неравенство:

$$
\left\langle p_{x}\left(\gamma_{1}, \eta, 0\right)+p_{x}\left(\gamma_{2}, \eta, 0\right), \eta\right\rangle+\rho(\eta) \geqslant 0
$$

где $\rho(\eta)=1$ и $p_{x}\left(\gamma_{k}, \eta, 0\right)=p_{x P_{i_{k}}}(\eta, 0)=p_{1}(\eta-0)=p_{1}(\eta)-$ субградиент $p(\eta)$, вычисленный в точке $w=\left(x-P_{i_{k}}\right) /\left\|P_{i_{k}}-x\right\|, k=1,2$.

Оказьвается, во всех имеющихся случаях предыдущее неравенство эквивалентно одному и тому же неравенству

$$
i_{2}-i_{1} \geqslant \frac{m}{3}-1
$$

Неравенство (1) влечет, что среди сетей Г с максимально возможной степенью вершины $O$ существует сеть, у которой все отличные от $O$ граничные вершины являются вершинами из $\Sigma$. Поэтому для получения ограничения на возможные степени вершины $O$ сети $Г$ достаточно рассмотреть случай, когда ребра из Г соединяют начало координат и вершины $m$-угольника $\Sigma$. Пусть $\Gamma$ - такая сеть, степень вершины $O$ равна $s$, и $\gamma_{j+1}=O P_{i_{j}}, j=0, \ldots, s-1,-$ последовательные при положительном обходе вершины $O$ ребра из $\Gamma$. Обозначим через $k_{j}$ количество вершин $m$-угольника $\Sigma$ между соседними ребрами с номерами $j$ и $j+1$. Найдем наименьшее $k_{j}$, при котором вьполняется неравенство (1). Имеем

a) $k_{j}=2 n / 3-2$, если $2 n \equiv 0(\bmod 3)$;

b) $k_{j}=(2 n-1) / 3-1$, если $2 n \equiv 1(\bmod 3)$;

c) $k_{j}=(2 n-2) / 3-1$, если $2 n \equiv 2(\bmod 3)$.

Найдем теперь ограничение на $s$ в каждом из трех случаев.

a) Пусть $2 n \equiv 0(\bmod 3)$. Так как количество вершин $m$-угольника $\Sigma$ равно $2 n$, получаем $s(2 n / 3-2)+s \leqslant 2 n$, откуда $s \leqslant 3+9 /(2 n-3)$. Функция $9 /(2 n-3)$ монотонно убывает. Отсюда следует, что степень вершины $O$ при $m=6$ меньше или равна 6 , при $m=12$ - меньше или равна 4 , при остальных $m$ - меньше или равна 3 .

b) Пусть $2 n \equiv 1(\bmod 3)$; тогда $s((2 n-1) / 3-1)+s \leqslant 2 n$, откуда $s \leqslant 3+3 /(2 n-1)$. Поэтому степень вершины $O$ при $m=4$ меньше или равна 4 , при остальных $m$ - меньше или равна 3.

c) Пусть $2 n \equiv 2(\bmod 3) ;$ тогда $s((2 n-2) / 3-1)+s \leqslant 2 n$, откуда $s \leqslant 3+6 /(2 n-2)$. Поэтому степень вершины $O$ при $m=8$ меньше или равна 4 , при остальных $m$ - меньше или равна 3.

Оказьвается, что в случае, когда $m=6$ и вершина $O$ - внутренняя, полученную оценку $s \leqslant 6$ можно усилить. Пусть $\Gamma$ - такая сеть. Мы покажем, что $s$ не может быть равна 5 и 6 . Поэтому $s \leqslant 4$, что и завершает доказательство теоремы.

Пусть $s=6$. Из неравенств (1) следует, что возможен единственный вариант, а именно, когда сеть $Г$ состоит из 6 ребер $\gamma_{k+1}=O P_{k}, k=0, \ldots, 5$.

Рассмотрим тип расщепления $\Gamma^{\prime}$ сети $\Gamma$ с единственной вырожденной компонентой $H$, образ которой при отображении $\Gamma^{\prime}$ совпадает с $O$ и которая представляет собой путь $x_{1} x_{2}, x_{2} x_{3}, x_{3} x_{4}$, причем вершина $x_{1}$ инцидентна ребрам $\gamma_{5}$ и $\gamma_{6}$, вершина $x_{2}$ инцидентна ребру $\gamma_{4}$, вершина $x_{3}$ инцидентна ребру $\gamma_{3}$, а вершина $x_{4}$ - ребрам $\gamma_{1}$ и $\gamma_{2}$. Рассмотрим деформацию сети $\Gamma^{\prime}$ в классе линейных сетей, при которой вершины $x_{i}$ равномерно движутся по прямым, причем $x_{1}$ движется со скоростью $\eta_{1}=(-1 / 2,-\sqrt{3} / 2)$, вершина $x_{2}$ - со скоростью $\eta_{2}=(-1,0)$, вершина $x_{3}-$ со скоростью $\eta_{3}=(-1 / 2, \sqrt{3} / 2)$, и вершина 
$x_{4}-$ со скоростью $\eta_{4}=(1 / 2, \sqrt{3} / 2)$. Отметим, что векторы $\eta_{i}$ имеют единичную норму. В соответствии с теоремой 3.1 , так как выражение

$$
\begin{aligned}
& \left\langle p_{x_{1}}\left(\gamma_{5}, \eta_{1}, 0\right)+p_{x_{1}}\left(\gamma_{6}, \eta_{1}, 0\right), \eta_{1}\right\rangle+\left\langle p_{x_{2}}\left(\gamma_{4}, \eta_{2}, 0\right), \eta_{2}\right\rangle+\left\langle p_{x_{3}}\left(\gamma_{3}, \eta_{3}, 0\right), \eta_{3}\right\rangle \\
& \quad+\left\langle p_{x_{4}}\left(\gamma_{1}, \eta_{4}, 0\right)+p_{x_{4}}\left(\gamma_{2}, \eta_{4}, 0\right), \eta_{4}\right\rangle+\rho\left(\eta_{1}-\eta_{2}\right)+\rho\left(\eta_{2}-\eta_{3}\right)+\rho\left(\eta_{3}-\eta_{4}\right) \\
& =0-1-1-1+0-1+1+1+1=-1
\end{aligned}
$$

отрицательно, тип расшепления $\Gamma^{\prime}$ не является слабо экстремальной сетью, а значит, и сеть $Г$ не экстремальна.

Пусть $s=5$. Из неравенств (1) следует, что возможны два, с точностью до поворота, варианта, а именно, когда сеть $Г$ состоит из 5 ребер $\gamma_{k+1}=O P_{k}, k=0, \ldots, 4$, или когда сеть Г состоит из 4 ребер $\gamma_{k+1}=O P_{k}, k=0, \ldots, 3$, и ребра $\gamma_{5}$, соединяющего точку $O$ и точку, лежащую на интервале $\left(P_{4}, P_{5}\right)$. Разберем первый случай (второй случай разбирается аналогично).

Рассмотрим тип расщепления $\Gamma^{\prime}$ сети $\Gamma$ с единственной вырожденной компонентой $H$, образ которой при отображении $\Gamma^{\prime}$ совпадает с $O$ и которая представляет собой путь $x_{1} x_{2}, x_{2} x_{3}$, причем вершина $x_{1}$ инцидентна ребрам $\gamma_{4}$ и $\gamma_{5}$, вершина $x_{2}$ инцидентна ребру $\gamma_{3}$, а вершина $x_{3}-$ ребрам $\gamma_{1}$ и $\gamma_{2}$. Рассмотрим деформацию сети $\Gamma^{\prime}$ в классе линейных сетей, при которой вершины $x_{i}$ равномерно движутся по прямым, причем $x_{1}$ движется со скоростью $\eta_{1}=(-1,0)$, вершина $x_{2}-$ со скоростью $\eta_{2}=(-1 / 2, \sqrt{3} / 2)$, и вершина $x_{3}$ - со скоростью $\eta_{3}=(1 / 2, \sqrt{3} / 2)$. Отметим, что векторы $\eta_{i}$ имеют единичную норму. В соответствии с теоремой 3.1, так как выражение

$$
\begin{aligned}
& \left\langle p_{x_{1}}\left(\gamma_{5}, \eta_{1}, 0\right)+p_{x_{1}}\left(\gamma_{4}, \eta_{1}, 0\right), \eta_{1}\right\rangle+\rho\left(\eta_{1}-\eta_{2}\right)+\left\langle p_{x_{2}}\left(\gamma_{3}, \eta_{2}, 0\right), \eta_{2}\right\rangle \\
& \quad+\rho\left(\eta_{2}-\eta_{3}\right)+\left\langle p_{x_{3}}\left(\gamma_{1}, \eta_{3}, 0\right)+p_{x_{3}}\left(\gamma_{2}, \eta_{3}, 0\right), \eta_{3}\right\rangle=0-1-1+0-1+1+1=-1
\end{aligned}
$$

отрицательно, тип расщепления $\Gamma^{\prime}$ не является слабо экстремальной сетью, а значит и сеть Г не экстремальна. Доказательство закончено.

Доказательство основной теоремы опирается на следующие шесть лемм, которые из-за недостатка места мы приведем без доказательства.

ЛЕмма 3.1. Если для двух ненулевых векторов $\eta_{1}$ и $\eta_{2}$ выполнены неравенства $\left\langle\theta, \eta_{1}\right\rangle \geqslant 0 u\left\langle\theta, \eta_{2}\right\rangle \geqslant 0$, где $\theta-$ фиксированныи ковектор, то неравенство $\langle\theta, \eta\rangle \geqslant 0$ выполнено для любого ненулевого вектора $\eta$, лехсашего внутри угла, образованного векторами $\eta_{1}$ и $\eta_{2}$.

ЛЕМмА 3.2. Пусть $\eta_{1}=a(\cos \varphi, \sin \varphi)$, и точка $\frac{a}{\rho\left(\eta_{1}\right)}(\cos \varphi, \sin \varphi)$ принадлежсит полуинтервалу $\left[P_{i}, P_{i+1}\right)$. Тогда

$$
\rho\left(\eta_{1}\right)=a \frac{\cos \left(\varphi-\frac{\pi}{n}\left(i+\frac{1}{2}\right)\right)}{\cos \frac{\pi}{2 n}}
$$


ЛЕмма 3.3. Пусть $\eta_{1}=a(\cos \varphi, \sin \varphi) u \eta_{2}=b(\cos \psi, \sin \psi)$. Тогдa

1) если $0 \leqslant \psi-\varphi \leqslant \pi$ или $\varphi-\psi \geqslant \pi$, mo

$$
\rho\left(\eta_{2}-\eta_{1}\right)=\frac{b \cos \left(\psi-\frac{\pi}{n}(i+1 / 2)\right)-a \cos \left(\varphi-\frac{\pi}{n}(i+1 / 2)\right)}{\cos (\pi / 2 n)}
$$

где точка $\left(\eta_{2}-\eta_{1}\right) / \rho\left(\eta_{2}-\eta_{1}\right)$ принадлежит полуинтервалу $\left[P_{i}, P_{i+1}\right)$;

2) если $0 \leqslant \varphi-\psi \leqslant \pi$ или $\psi-\varphi \geqslant \pi$, mo

$$
\rho\left(\eta_{1}-\eta_{2}\right)=\frac{a \cos \left(\varphi-\frac{\pi}{n}(i+1 / 2)\right)-b \cos \left(\psi-\frac{\pi}{n}(i+1 / 2)\right)}{\cos (\pi / 2 n)}
$$

әде точка $\left(\eta_{1}-\eta_{2}\right) / \rho\left(\eta_{1}-\eta_{2}\right)$ принадлежит полуинтервалу $\left[P_{i}, P_{i+1}\right)$.

Лемма 3.4. Пусть $m=6, \quad \eta_{1}=a(\cos \varphi, \sin \varphi), \quad \eta_{2}=b(\cos \psi, \sin \psi)$, əде $\varphi \in$ $[2 \pi / 3, \pi], \quad \psi \in[-\pi / 3, \pi / 3]$. Тогда $\rho\left(\eta_{1}-\eta_{2}\right)$ больше или равно $\rho\left(\eta_{1}\right)$ и $\rho\left(\eta_{2}\right)$.

Лемма 3.5. Пусть $m=6, \quad \eta_{1}=a(\cos \varphi, \sin \varphi), \quad \eta_{2}=b(\cos \psi, \sin \psi)$, әде $\varphi \in$ $[\pi / 2,2 \pi / 3], \psi \in[0, \pi / 3]$. Тогда $\left\langle p_{O P_{3}}\left(\eta_{1}, 0\right)+p_{O P_{2}}\left(\eta_{1}, 0\right), \eta_{1}\right\rangle+\rho\left(\eta_{1}-\eta_{2}\right) \geqslant 0$.

Лемма 3.6. Пусть $m=6, \quad \eta_{1}=a(\cos \varphi, \sin \varphi), \quad \eta_{2}=b(\cos \psi, \sin \psi)$, əде $\varphi \in$ $[2 \pi / 3, \pi], \quad \psi \in[\pi, 4 \pi / 3]$. Тогда $\left\langle p_{O P_{2}}\left(\eta_{1}, 0\right), \eta_{1}\right\rangle+\rho\left(\eta_{1}\right)+\rho\left(\eta_{2}-\eta_{1}\right) \geqslant \rho\left(\eta_{2}\right)$.

ДОКАЗАТЕЛЬСТВО ОСНОВНОЙ ТЕОРЕМЫ. Пусть $Г$ - вложенная локально минимальная сеть и $x$ - произвольная ее вершина. Покажем, что тип вершины $x$ описьвается теоремой. Пусть $\Gamma_{\text {loc }}(x)$ - локальная сеть с центром в $x$ для $\Gamma$. По определению сеть $\Gamma_{\text {loc }}(x)$ кратчайшая. Так как сдвиги не меняют экстремальные свойства сети, без ограничения общности будем предполагать, что $x$ находится в начале координат. В силу утверждений $3.1,3.2$ и неравенства (1) вершина $x$ сети $\Gamma_{\text {loc }}(x)$ имеет не превосходящую 6 степень и один из следующих типов:

1) если степень вершины $x$ равна 1 , то type $v(x)=\{[]\}$;

2) если степень вершины $x$ равна 2 , то при $2 n \equiv k(\bmod 3), k=1,2,3$, имеем $\operatorname{type}_{v}(x)=\{[],[] ; l\}$, причем $3-n \leqslant l \leqslant k$

3 ) если степень вершины $x$ равна 3 , то

(a) при $2 n \equiv 0(\bmod 3)$ имеем type ${ }_{v}(x)=\left\{[,[],[] ; 3,3\}\right.$ или $\operatorname{type}_{v}(x)=\{[],[], *$; $0,3\}$

(b) при $2 n \equiv k(\bmod 3), k=1,2$, имеем type ${ }_{v}(x)=\{*,[], * ; k, k\}$;

(c) при $2 n \equiv 2(\bmod 3)$ имеем type $v(x)=\{[],[], * ; 2,2\}$;

4) если степень вершины $x$ равна 4 , то $m=4,6,8$ или 12 , и

(a) при $m=4,8$ или 12 имеем type ${ }_{v}(x)=\{*, *, *, * ; m / 4, m / 4, m / 4\}$;

(b) при $m=6$ имеем $\operatorname{type}_{v}(x)=\{*,[], *,[] ; 3,3,3\}$ или $\operatorname{type}_{v}(x)=\{[],[], *, *$; $3,3,3\}$;

5 ) если степень вершины $x$ равна 5 или 6 , то эта вершина граничная, $m=6$ и

(a) если степень вершины $x$ равна 5 , то type $v(x)=\{[], *, *, *, * ; 3,3,3,3\}$;

(b) если степень вершины $x$ равна 6 , то type $v(x)=\{*, *, *, *, *, * ; 3,3,3,3,3\}$.

Остается доказать, что вершина $x$ не может иметь некоторые из вьшеперечисленных типов. Для каждого типа, перечисленного вьше, который не входит в утверждение основной теоремы, легко можно указать базовьй тип расщепления $\Gamma_{\text {loc }}^{\prime}(x): G_{\text {loc }}^{\prime}(x) \rightarrow \mathbb{R}^{2}$ 
и отображение $\eta: V_{G_{\text {loc }}^{\prime}(x)} \rightarrow \mathbb{R}^{2}$, где $\eta\left(\partial G_{\text {loc }}^{\prime}(x)\right)=0$, такие, что $\Gamma_{\text {loc }}^{\prime}(x)$ в силу теоремы 3.1 не является слабо экстремальной сетью. Последнее в силу утверждения 3.1 противоречит тому, что сеть $\Gamma_{\text {loc }}(x)$ кратчайшая.

Докажем обратное утверждение. Пусть вершина $x$ сети Г имеет один из перечисленных в утверждении основной теоремы типов и, как вьше, находится в начале координат $O$. Для завершения доказательства теоремы достаточно показать, что $\Gamma_{\text {loc }}(x)-$ кратчайшая сеть. Для этого в силу теоремы 3.2 и утверждения 3.1 достаточно рассмотреть все базовые типы расщепления $\Gamma_{\text {loc }}^{\prime}(x)$ сети $\Gamma_{\text {loc }}(x)$ и проверить их на слабую экстремальность. Дальнейшее доказательство будет следовать из теоремы 3.1 и из лемм 3.1-3.6.

Из-за недостатка места мы рассмотрим, например, случай, когда единичная окружность $\Sigma$ является 6 -угольником и степень вершины $x$ равна 5 . Будем считать, что случаи, когда $x$ имеет степень меньшую 5 , уже разобраны. Пусть сеть $\Gamma_{\text {loc }}(x)$ состоит из 5 ребер $\gamma_{1}=O P_{0}, \gamma_{2}=O P$ и $\gamma_{k}=O P_{k}, k=3, \ldots, 5$, где $P_{k}$ - вершина 6-угольника с номером $k$, и точка $P$ лежит на интервале $\left(P_{1}, P_{2}\right)$.

Из-за большого числа базовых типов расшеплений ограничимся рассмотрением лиш одного из них и проверим его на слабую экстремальность. Пусть базовьци тип расщепления $\Gamma_{\mathrm{loc}}^{\prime}(x): G_{\mathrm{loc}}^{\prime}(x) \rightarrow \mathbb{R}^{2}$ сети $\Gamma_{\text {loc }}(x)$ представляет собой сеть с единственной вырожденной компонентой $H^{\prime}$, образ которой при отображении $\Gamma_{\text {loc }}^{\prime}(x)$ совпадает с $O$ и которая представляет собой путь $x_{1} x_{2}, x_{2} x_{3}, x_{3} x_{4}, x_{4} x_{5}$, причем вершины $x_{2}, x_{3}$ и $x_{4}$ инцидентны ребрам $\gamma_{3}, \gamma_{2}$ и $\gamma_{4}$ соответственно, вершина $x_{5}$ инцидентна ребрам $\gamma_{1}$ и $\gamma_{5}$, а вершина $x_{1}$ граничная.

По теореме 3.1 слабая экстремальность сети $\Gamma_{\mathrm{loc}}^{\prime}(x)$ равносильна следующему условию: для каждого отображения $\eta: V_{G_{\text {loc }}^{\prime}(x)} \rightarrow \mathbb{R}^{2}$, где $\eta\left(\partial G_{\text {loc }}^{\prime}(x)\right)=0$, имеет место неравенство:

$$
\begin{aligned}
\rho\left(\eta\left(x_{2}\right)\right)+ & \left\langle p_{x_{2}}\left(\gamma_{3}, \eta\left(x_{2}\right), 0\right), \eta\left(x_{2}\right)\right\rangle+\rho\left(\eta\left(x_{2}\right)-\eta\left(x_{3}\right)\right) \\
& +\left\langle p_{x_{3}}\left(\gamma_{2}, \eta\left(x_{3}\right), 0\right), \eta\left(x_{3}\right)\right\rangle+\rho\left(\eta\left(x_{3}\right)-\eta\left(x_{4}\right)\right)+\left\langle p_{x_{4}}\left(\gamma_{4}, \eta\left(x_{4}\right), 0\right), \eta\left(x_{4}\right)\right\rangle \\
& +\rho\left(\eta\left(x_{4}\right)-\eta\left(x_{5}\right)\right)+\left\langle p_{x_{5}}\left(\gamma_{1}, \eta\left(x_{5}\right), 0\right)+p_{x_{5}}\left(\gamma_{5}, \eta\left(x_{5}\right), 0\right), \eta\left(x_{5}\right)\right\rangle \geqslant 0 .
\end{aligned}
$$

Если $\eta\left(x_{2}\right)$ лежит в угле от $-2 \pi / 3$ до $2 \pi / 3$, т.е. $\left\langle p_{x_{2}}\left(\gamma_{3}, \eta\left(x_{2}\right), 0\right), \eta\left(x_{2}\right)\right\rangle \geqslant 0$, используя неравенство $\rho\left(\eta\left(x_{2}\right)\right)+\rho\left(\eta\left(x_{3}\right)-\eta\left(x_{2}\right)\right) \geqslant \rho\left(\eta\left(x_{3}\right)\right)$, мы получим, что неравенство (2) вытекает из неравенства

$$
\begin{aligned}
\rho\left(\eta\left(x_{3}\right)\right)+ & \left\langle p_{x_{3}}\left(\gamma_{2}, \eta\left(x_{3}\right), 0\right), \eta\left(x_{3}\right)\right\rangle+\rho\left(\eta\left(x_{3}\right)-\eta\left(x_{4}\right)\right)+\left\langle p_{x_{4}}\left(\gamma_{4}, \eta\left(x_{4}\right), 0\right), \eta\left(x_{4}\right)\right\rangle \\
& +\rho\left(\eta\left(x_{4}\right)-\eta\left(x_{5}\right)\right)+\left\langle p_{x_{5}}\left(\gamma_{1}, \eta\left(x_{5}\right), 0\right)+p_{x_{5}}\left(\gamma_{5}, \eta\left(x_{5}\right), 0\right), \eta\left(x_{5}\right)\right\rangle \geqslant 0
\end{aligned}
$$

которое верно, так как сеть с граничной вершиной $x$ и с ребрами $\gamma_{1}, \gamma_{2}, \gamma_{4}$ и $\gamma_{5}$ имеет $\operatorname{type}_{v}(x)=\{*,(), *, * ; 3,0,3\}$, являющийся частным случаем типа из пункта $4(\mathrm{c})$ основной теоремы, которьй уже разобран в силу предположения. Поэтому нам остается рассмотреть случай, когда $\eta\left(x_{2}\right)$ лежит в угле от $2 \pi / 3$ до $4 \pi / 3$. Аналогично доказывается, что достаточно рассмотреть лишь те $\eta\left(x_{3}\right)$, которые лежат в угле от 0 до $\pi$, те $\eta\left(x_{4}\right)$, которые лежат в угле от $\pi$ до $5 \pi / 3$ и те $\eta\left(x_{5}\right)$, которые лежат в угле от $-\pi / 2$ до $\pi / 6$.

Рассмотрим два случая, которые и завершат доказательство основной теоремы. 
1) $\eta\left(x_{4}\right)$ лежит в угле от $\pi$ до $4 \pi / 3, \eta\left(x_{2}\right)$ лежит в угле от $2 \pi / 3$ до $4 \pi / 3, \eta\left(x_{3}\right)$ лежит в угле от 0 до $\pi, \eta\left(x_{5}\right)$ лежит в угле от $-\pi / 2$ до $\pi / 6$. Неравенство $(2)$ в силу лемм 3.4 и 3.5 вытекает из неравенства

$$
\begin{aligned}
\rho\left(\eta\left(x_{2}\right)\right)+ & \left\langle p_{x_{2}}\left(\gamma_{3}, \eta\left(x_{2}\right), 0\right), \eta\left(x_{2}\right)\right\rangle+\rho\left(\eta\left(x_{2}\right)-\eta\left(x_{3}\right)\right) \\
& +\left\langle p_{x_{3}}\left(\gamma_{2}, \eta\left(x_{3}\right), 0\right), \eta\left(x_{3}\right)\right\rangle+\rho\left(\eta\left(x_{3}\right)-\eta\left(x_{4}\right)\right)+\left\langle p_{x_{4}}\left(\gamma_{4}, \eta\left(x_{4}\right), 0\right), \eta\left(x_{4}\right)\right\rangle \geqslant 0
\end{aligned}
$$

которое верно, так как сеть с граничной вершиной $x$ и с ребрами $\gamma_{2}, \gamma_{3}$ и $\gamma_{4}$ имеет $\operatorname{type}_{v}(x)=\{(), *, * ; 3,3\}$, являющийся частньм случаем типа из пункта $3(\mathrm{c})$ основной теоремы, которьй уже разобран в силу предположения.

2) $\eta\left(x_{4}\right)$ лежит в угле от $4 \pi / 3$ до $5 \pi / 3, \eta\left(x_{2}\right)$ лежит в угле от $2 \pi / 3$ до $4 \pi / 3, \eta\left(x_{3}\right)$ лежит в угле от 0 до $\pi, \eta\left(x_{5}\right)$ лежит в угле от $-\pi / 2$ до $\pi / 6$. Неравенство $(2)$ в силу леммы 3.4 вытекает из следующих двух неравенств:

$$
\begin{aligned}
\rho\left(\eta\left(x_{2}\right)\right)+ & \left\langle p_{x_{2}}\left(\gamma_{3}, \eta\left(x_{2}\right), 0\right), \eta\left(x_{2}\right)\right\rangle+\rho\left(\eta\left(x_{2}\right)-\eta\left(x_{3}\right)\right)+\left\langle p_{x_{3}}\left(\gamma_{2}, \eta\left(x_{3}\right), 0\right), \eta\left(x_{3}\right)\right\rangle \geqslant 0, \\
\rho\left(\eta\left(x_{4}\right)\right)+ & \left\langle p_{x_{4}}\left(\gamma_{4}, \eta\left(x_{4}\right), 0\right), \eta\left(x_{4}\right)\right\rangle+\rho\left(\eta\left(x_{4}\right)-\eta\left(x_{5}\right)\right) \\
& +\left\langle p_{x_{5}}\left(\gamma_{1}, \eta\left(x_{5}\right), 0\right)+p_{x_{5}}\left(\gamma_{5}, \eta\left(x_{5}\right), 0\right), \eta\left(x_{5}\right)\right\rangle \geqslant 0 .
\end{aligned}
$$

Первое неравенство верно из-за того, что сеть с граничной вершиной $x$ и с ребрами $\gamma_{2}$ и $\gamma_{3}$ имеет type ${ }_{v}(x)=\{(), * ; 3\}$, являющийся частным случаем типа из пункта $2(\mathrm{~b})$ основной теоремы, которьй уже разобран в силу предположения, а второе из-за того, что сеть с граничной вершиной $x$ и с ребрами $\gamma_{1}, \gamma_{4}$ и $\gamma_{5}$ имеет type $v(x)=\{*, *, * ;-6,3\}$, являющийся частным случаем типа из пункта $3(\mathrm{c})$ основной теоремы, которьй также уже разобран в силу предположения. Доказательство основной теоремы закончено.

\section{СПИСОК ЦИТИРОВАННОЙ ЛИТЕРАТУРЫ}

[1] Du D. Z., Hwang F. K., Weng J. F. Steiner minimal trees for Regular Polygons // Disk. and Comp. Geometry. 1987. V. 2. P. 65-84.

[2] Fermat P. Abhandlungen uber Maxima und Minima // Oswalds, Klassiker der Exakten Wissenschaften. № 238, 1934.

[3] Jarnik V., Kössler M. O minimalnich grafeth obeahujiicich n danijch bodu // Cas. Pest. Mat. a Fys. 1934. V. 63. P. 223-235.

[4] Smith W. D. How to find Steiner minimal trees in Euclidean $d$-space // Algoritmica. 1992. № 7. P. 137-177.

[5] Francis R. L. A note on the optimum location of new machines in existing plant loyouts // J. Indust. Engrg. 1963. V. 14. P. 57-59.

[6] Hanan M. On Steiner's Problem with Rectilinear Distance // SIAM J. Appl. Math. 1966. V. 14. P. 255-265.

[7] Hwang F. K. On Steiner minimal trees with rectilinear distance // SIAM J. Appl. Math. 1976. V. 30. P. 104-114.

[8] Garey M. R., Johnson D. S. The Rectilinear Steiner Problem is NP-Complete. // SIAM J. Appl. Math. 1977. V. 32. P. 826-834.

[9] Ivanov A. O., Tuzhilin A. A. Branching Solutions to One-Dimensional Variational Problems. Singapore: World Scientific Publishing Co. Pte. Ltd, 2001. 